\title{
Cerebral Venous Thrombosis Developing after COVID-19 Vaccination: VITT, VATT, TTS, and More
}

\author{
Giuseppe Lippi, MD ${ }^{1}$ Emmanuel J. Favaloro, PhD, FFSc (RCPA) $2,3(1$ \\ 1 Section of Clinical Biochemistry, University of Verona, Verona, Italy \\ ${ }^{2}$ Department of Haematology, Institute of Clinical Pathology and \\ Medical Research (ICPMR), NSW Health Pathology, Westmead \\ Address for correspondence Giuseppe Lippi, MD, Section of Clinical \\ Hospital, Westmead, New South Wales, Australia \\ ${ }^{3}$ School of Biomedical Sciences, Charles Sturt University, Wagga \\ Wagga, New South Wales, Australia \\ Biochemistry, University of Verona, Verona 37126, Italy \\ (e-mail: giuseppe.lippi@univr.it). \\ Semin Thromb Hemost 2022;48:8-14.
}

\begin{abstract}
Keywords

- SARS-CoV-2

- COVID-19

- platelets

- thrombosis

- vaccine

Despite the huge efforts globally underway for preventing or limiting the spread of severe acute respiratory coronavirus disease 2 (SARS-CoV-2), the coronavirus disease 2019 (COVID-19) pandemic outbreak appears still virtually unstoppable. As for many other infectious diseases, COVID-19 vaccination has now become crucial for limiting viral spread, especially for averting hospitalizations, need for intensive care, and fatal outcome. Nonetheless, as for other vaccines, COVID-19 vaccination is not completely free from side effects. Among the adverse events that have been reported after receiving COVID-19 vaccination, special emphasis has been given to an unexpected number of thrombocytopenic episodes with or without thrombotic complications, especially in recipients of adenovirus-based COVID-19 vaccines. Along with a specific clinical presentation, encompassing "atypical" thrombosis (especially cerebral venous [sinus] thrombosis, CVT) more prevalent in young female subjects, this new syndrome called vaccine-induced thrombocytopenia and thrombosis (VITT) is characterized by, and thereby diagnosed for, the presence of three paradigmatic laboratory abnormalities, i.e., low platelet count $\left(<150 \times 10^{9} / \mathrm{L}\right)$, elevated plasma D-dimer levels $(>0.5 \mathrm{mg} / \mathrm{L})$, accompanied by a positive test for anti-PF4 (platelet factor 4) antibodies assayed with ELISA (enzyme-linked immunosorbent assay) techniques. Timely identification of these important abnormalities by both clinicians and laboratory professional is essential for early diagnosis and management of VITT, since the outcome of this condition may be fatal in half or even more of effected patients with severe disease. Therefore, this narrative review aims to review here the epidemiology, pathogenesis, clinical, and laboratory characteristics of VITT and other COVID-19 vaccine-associated thrombocytopenias.
\end{abstract}

Despite huge efforts underway globally for preventing or limiting the spread of severe acute respiratory coronavirus disease 2 (SARS-CoV-2), the ongoing coronavirus disease 2019 (COVID-19) pandemic outbreak still appears virtually unstoppable. As already seen for many other infectious diseases, ${ }^{1}$ COVID-19 vaccination is a cornerstone and a mainstay for limiting viral spread, especially for averting

published online October 25, 2021
Issue Theme Maintaining Hemostasis and Preventing Thrombosis in COVID-19-Part III; Guest Editors: Emmanuel J. Favaloro, PhD, FFSc (RCPA) and Giuseppi Lippi, MD hospitalizations, need for intensive care, and deaths worldwide. ${ }^{2}$ Nonetheless, as for most other therapies, vaccination is not completely free from side effects and even adverse events, which are typically classified in terms of location (local, systemic) and severity (mild, modest, and severe). ${ }^{3}$

The sudden emergence of cases of atypical thromboses in close proximity to COVID-19 vaccination was first reported (c) 2021. Thieme. All rights reserved. Thieme Medical Publishers, Inc., 333 Seventh Avenue, 18th Floor, New York, NY 10001, USA
DOI https://doi.org/ 10.1055/s-0041-1736168. ISSN 0094-6176. 
Table 1 Leading clinical and laboratory features of vaccine-induced thrombocytopenia and thrombosis (VITT) in recipients of adenovirus-based COVID-19 (coronavirus disease 2019) vaccines

\begin{tabular}{|l|l|}
\hline Characteristic & Description \\
\hline Patients & Mostly young individuals (aged $\leq 60 \mathrm{y}$ ); potential predominance of women \\
\hline Onset & $1-3$ wk after receiving adenovirus-based COVID-19 vaccines \\
\hline Thrombosis & $\begin{array}{l}\text { Mostly cerebral venous (sinus) thrombosis, occasionally accompanied by sys- } \\
\text { temic venous (especially splanchnic and pulmonary) and arterial thrombosis }\end{array}$ \\
\hline Pro-thrombotic conditions & Not evident in the majority of cases \\
\hline Laboratory data & $\begin{array}{l}\text { Low platelet count }\left(<150 \times 10^{9} / \mathrm{L}\right) \\
\text { Elevated plasma D-dimer level }(>0.5 \mathrm{mg} / \mathrm{L}) \\
\text { Positive anti-PF4 antibodies ELISA }\end{array}$ \\
\hline
\end{tabular}

Abbreviations: COVID-19, coronavirus disease 2019; PF4, platelet factor 4.

almost simultaneously by Greinacher et $\mathrm{al}^{4}$ and Schultz et al. $^{5}$ Both publications reported an unexpectedly high burden of cerebral venous thrombosis (CVT) in prevalently young women who received the AstraZeneca ChAdOx1 (Vaxzevria) vaccine. These case series (11 and 5, respectively) represented both high mortality (55 and 60\%, respectively) and morbidity. A review of cases as of the end of May, 2021, identified a case fatality of nearly $40 \%$ (31/79) in subsequent publications. $^{6}$

A plethora of overlapping definitions for this syndrome has since been provided by different organizations including TTS (thrombotic thrombocytopenia syndrome) and VATT (vaccine associated thrombosis with thrombocytopenia), ${ }^{6}$ although the term "vaccine-induced immune thrombotic thrombocytopenia" (VITT) seems to have been primarily selected, given that the key elements include acute thrombosis, thrombocytopenia (usually with platelet count $\left.<150 \times 10^{9} / \mathrm{L}\right)$, elevated $\mathrm{D}$-dimer values along with a positive ELISA (enzyme-linked immunosorbent assay) test for platelet factor 4 antibodies, all developing soon after COVID-19 vaccination (typically within $15-20$ days) $)^{7}$ (- Table $\mathbf{1}$ ).

Once potential causes of pseudothrombocytopenia have been ruled out, ${ }^{8}$ the combination of thrombosis, thrombocytopenia, and raised D-dimers, occurring soon after COVID-19 vaccination, is the key for identifying these patients as potentially having an episode of VITT. The clinical signs and symptoms of CVT mostly include the onset of new and severe headache, which cannot be alleviated by usual analgesics, often accompanied by blurred vision, nausea or vomiting, dysarthria, weakness, drowsiness or even seizures. ${ }^{9}$ Some patients may also have additional symptoms, such as shortness of breath, chest or abdominal pain and leg swelling, potentially associated with additional thromboses such as pulmonary thrombosis, splanchnic vein thrombosis, and deep vein thrombosis.

The potentially catastrophic picture of VITT has been welldescribed in a post-mortem analysis of two VITT cases by Pomara et al. ${ }^{10}$ The main features in both cases were massive thrombosis that especially involved the portal, splenic, and superior mesenteric veins in one case and the superior sagittal sinus in the other. Microscopic findings revealed the presence of vascular thrombotic occlusions within the microcirculation of multiple organs, with concomitant pres- ence of inflammatory infiltrates. Platelet aggregates were also found to diffusely lining the endothelial layer of small and medium size vessels, with signs of platelet phagocytosis by myeloid elements in the vascular spaces. The autopsies of two additional patients who died with VITT, and autopsies performed by Althaus et $\mathrm{al}^{11}$ demonstrated the presence of both arterial and venous thromboses in various organs, with complete thrombotic obstruction of cerebral sinuses, bilateral pulmonary embolism, occlusion of glomerular capillaries by hyaline thrombi containing fibrin and platelets.

\section{Epidemiology of VITT and Other Vaccine Associated Thrombocytopenias}

Although the epidemiological picture of VITT remains largely undefined, the frequency of both vaccine-associated thrombocytopenia and vaccine-associated idiopathic thrombocytopenic purpura (ITP) has been assessed in a Scottish populationbased study, including people who received both adenovirusbased AstraZeneca and mRNA-based Pfizer vaccines. ${ }^{12}$ The cumulative risk of developing thrombocytopenia was found to be significantly higher (relative risk, $2.8 ; 95 \% \mathrm{CI}, 1.39-5.67$ ) in recipients of AstraZeneca (but not Pfizer) vaccine than in nonvaccinated individuals 0 to 6 days after vaccination, while that of developing ITP was between 4.6 and 14.1, higher again in recipients of AstraZeneca (but not Pfizer) vaccine than in nonvaccinated individuals between 7 and 27 days after vaccination. Overall, the incidence rate of ITP was 1.13 (95\% CI; 0.621.63) per 100,000 AstraZeneca vaccine doses. With regard to the risk of thrombosis, an excess of both venous with CVT (between +19 and +71 ) and arterial (between +11 and +232 ) thrombotic events was recorded in subjects aged 16 to 59 years who received the AstraZeneca vaccine, while such excess risk was not evident in older subjects as well as in all those receiving the Pfizer vaccine. No substantial variation in the burden of hemorrhagic events could be found in all recipients of both vaccines.

A recent analysis of European data concluded that the risk of developing thrombocytopenia in recipients of AstraZeneca vaccine was 151 per million doses (compared with 33 per million doses in those receiving Pfizer vaccine), while that of developing cerebral/splanchnic thrombotic episodes 
or deaths was respectively 30 and 5 per million doses (compared with 4 and 0.4 per million doses in those receiving Pfizer vaccine). ${ }^{13}$ Additional important evidence on VITT has been provided in another study, which examined a series of 62 vascular cerebrovascular adverse events diagnosed in Germany in close proximity with COVID-19 vaccination, 45 of which were CVT. ${ }^{14}$ Some important elements emerged. First, 53 events were recorded in patients who received AstraZeneca vaccine, nine in those who received Pfizer vaccine and none in those who received Moderna vaccine. Considering that the overall prevalence of CVT in the general population is typically comprised between 0.02 and 0.15 per 100,000 person-months, the incidence rate of CVT within the first month from administration of the first vaccine dose was estimated as being 0.55 (95\% CI, 0.38-0.78) per 100,000 person-months for all vaccines, increasing to 1.52 (95\% CI, 1.00-2.21) per 100,000 person-months for AstraZeneca, but being as low as 0.11 (95\% CI, 0.03-0.29) per 100,000 personmonths for Pfizer and 0.00 (95\% CI, 0.00-1.48) per 100,000 person-months for Moderna vaccines. The adjusted incidence rate ratio of AstraZeneca vaccine was nearly 10-fold higher (9.68; 95\% CI, 3.46-34.98) compared with mRNAbased vaccines, and approximately threefold higher (3.14; 95\% CI, 1.22-10.65) for women compared with men, while the trend of higher risk in subjects aged $<60$ years did not reach statistical significance $(2.14 ; 95 \% \mathrm{CI}, 0.83-6.78) .{ }^{14}$ It is therefore important to mention that cases of secondary immune thrombocytopenia (ITP) occurring in recipients of mRNA-based COVID-19 vaccines have been widely reported in the current scientific literature, ${ }^{15}$ though it remains to be determined whether this association is causal or merely coincidental.

Both currently approved adenovirus-based vaccines seem to be associated with this pathology, even if CVT episodes appear to be more frequently reported in recipients of AstraZeneca (3.6 per million doses; 99\% CI, 2.7-4.8 per million doses) than in those of Johnson \& Johnson vaccines (0.9 per million doses; $99 \% \mathrm{CI}, 0.2-2.3$ per million doses). ${ }^{16}$ An additional analysis of cases so far described has evidenced that there may be some important differences between the vaccine-induced thrombosis caused by either adenovirusbased vaccine. Compared with Johnson \& Johnson, the thrombotic syndrome observed in AstraZeneca recipients seems to develop earlier, with higher D-dimer values and higher likelihood of being positive for functional platelet activation testing, but with a lower risk of developing intracerebral bleeding. No other major clinical and laboratory differences could be seen, beside a higher propensity to develop internal jugular vein thrombosis in Johnson \& Johnson vaccine recipients. ${ }^{17}$ More recently, Krzywicka et al conducted a systematic analysis of all cases of post-COVID19 vaccination CVT reported to the EudraVigilance database of the European Medicines Agency (EMA). ${ }^{18}$ Overall, 213 reports could be found, $187(87.8 \%)$ in recipients of adenovirus-based AstraZeneca vaccine, 25 (11.7\%) in mRNA-based Pfizer vaccine recipients, and the remaining one $(0.5 \%)$ in a recipient of mRNA-based Moderna vaccine. Importantly, of all patients in whom the outcome had been reported, the death rate was 37.6\% (44/177) in recipients of adenovirusbased AstraZeneca vaccine compared with $20 \%(2 / 10)$ in recipients of either mRNA-based vaccine, thus considerably higher than that reported for cases of pre-COVID-19 CVT (3\%; 3/100).

Therefore, despite needing more time to garner a final epidemiological picture of CVT-associated VITT, especially because the metrics remain widely heterogeneous across different reports (epidemiologic data are referred to vaccine doses administered, recipients, person-months, etc.), it seems reasonable to conclude that this syndrome mostly involves younger individuals (i.e., aged 60 years or younger), potentially with a female predominance, develops within 1 to 3 weeks after receiving the first vaccine dose, and is indeed more prevalent after receiving an adenovirus-based COVID19 vaccine. Nonetheless, as recently highlighted by an expert consensus on vaccine-induced immune thrombotic thrombocytopenia, ${ }^{19}$ the risk of VITT remains substantially lower than that of dying from COVID-19, so that the favorable effects of vaccination largely offset the risk of developing severe forms and dying from COVID-19, even at a younger age (i.e., the risk of VITT is $18-21$ per million vaccine recipients compared with a risk of dying for COVID-19 up to 100 per million people aged 20-49 years in areas with high prevalence of SARS-CoV-2 infections).

\section{Pathogenesis of VITT}

The pathogenesis of CVT has been recently reviewed by Ulivi et al. ${ }^{20}$ Briefly, this pathology, which is typically caused by occlusion of cerebral venous sinuses (i.e., cerebral venous sinus thrombosis) or smaller cortical veins (i.e., cortical vein thrombosis), mostly develops in young females (in the third decade of age, with 2:1 female:male ratio). The use of estrogen-containing oral contraceptives is the most prevalent risk factor for CVT, associated with a prothrombotic condition (either inherited or acquired) in the vast majority of patients. The most frequent thrombophilic conditions are factor V Leiden, prothrombin gene polymorphism G20210A, along with deficiencies of physiological inhibitors of blood coagulation (i.e., protein $\mathrm{C}$ or $\mathrm{S}$, antithrombin). With regard to the acquired conditions other than oral contraceptives, an increased burden of antiphospholipid antibody syndrome, obesity, cancer, local infections, and head trauma has been reported, while higher frequency is also seen during pregnancy and puerperium. Although the prognosis is mostly favorable in the vast majority of patients with CVT, around $15 \%$ of them may have long-term consequences or even die of its consequences.

What has clearly emerged since the description of the first cases, is that the CVT developing in patients with VITT seems to be different in terms of pathogenesis and evolution from its more traditional form. The clearest evidence, as originally underpinned by Greinacher et $\mathrm{al}^{4}$ and Schultz et $\mathrm{al}^{5}{ }^{5}$ is that no clear prothrombotic risk factors were present. Of the five VITT patients described by Schultz et al, four (80\%) were female, one was using oral contraceptives, one contraceptive vaginal ring and one hormone-replacement therapy, though 


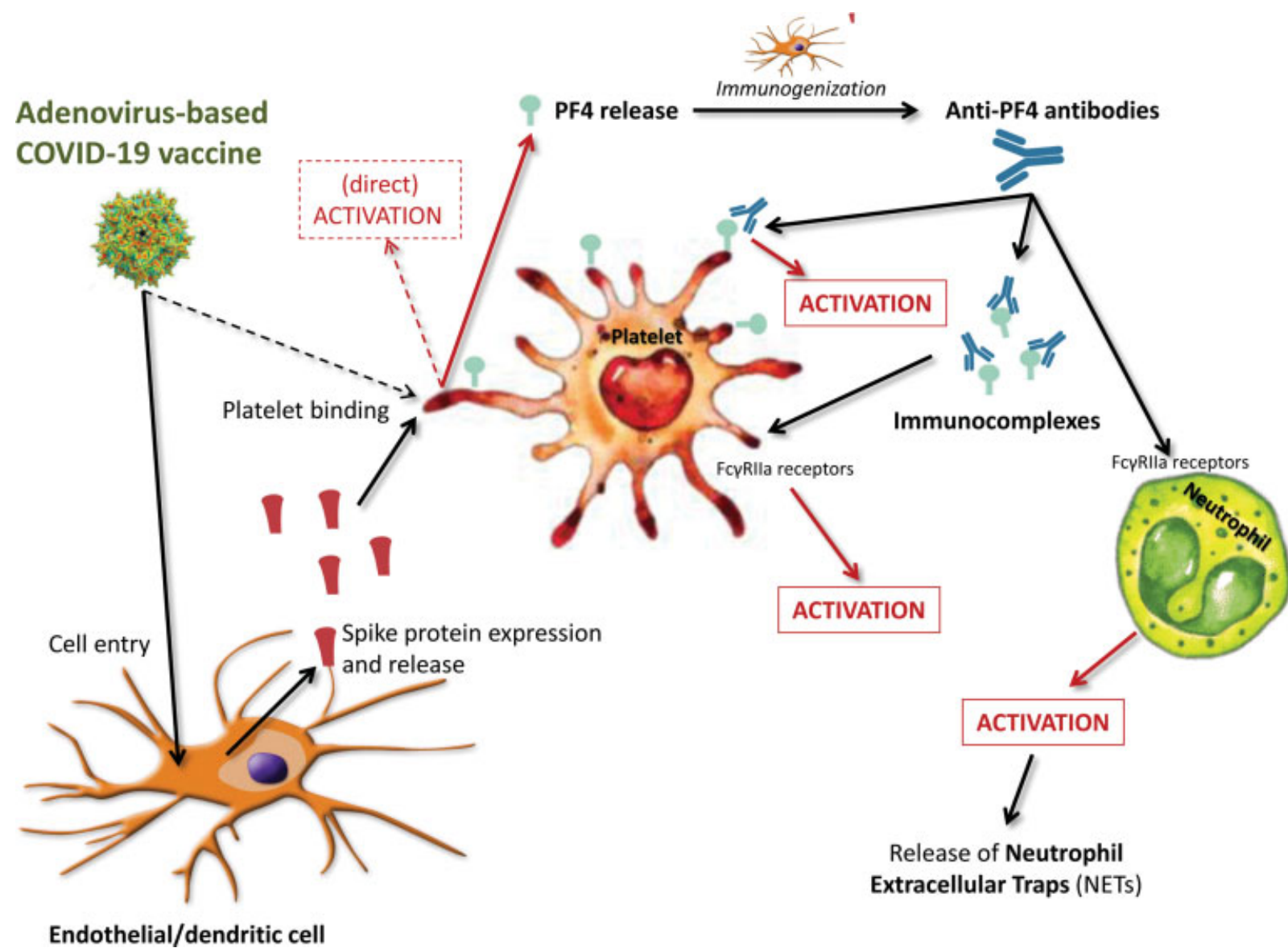

Fig. 1 Pathogenetic mechanisms potentially involved in vaccine-induced thrombocytopenia and thrombosis in recipients of adenovirus-based COVID-19 (coronavirus disease 2019) vaccines. PF4, platelet factor 4.

all were found to have thrombocytopenia (between 10$\left.70 \times 10^{9} / \mathrm{L}\right)$, elevated $\mathrm{D}$-dimer values $(>13 \mathrm{mg} / \mathrm{L})$, along with high values of IgG antibodies against PF4-polyanion complexes. None had known pre-existing prothrombotic conditions and three $(60 \%)$ of these patients had fatal outcome. Similarly, in only one of the 11 VITT cases described by Greinacher et al was a pre-existing pro-thrombotic condition able to be identified (FVL), while in all in whom a PF4antibody ELISA test was performed, the result was positive. All patients had thrombocytopenia (between 8 and $107 \times 10^{9} / \mathrm{L}$ ) and elevated D-dimer values (between 1.8 and $142 \mathrm{mg} / \mathrm{L}$ ). Importantly, 6/11 (55\%) of these patients had fatal outcome, and almost all samples tested from a further series of patients with suspected VITT (22/24; 92\%) displayed platelet activation after challenge with PF4. Similar evidence was provided in a 12, U.S.-based, case series of adenovirus-based (Johnson \& Johnson) COVID-19 vaccine recipients, as reported by See and co-authors. ${ }^{21}$ Overall, use of oral contraceptives was reported in only one of the patients (six were obese), and only one could be safely discharged at home. As now could be predicted, all patients tested had thrombocytopenia (between 9 and $127 \times 10^{9} / \mathrm{L}$ ), elevated D-dimer values (between 1.1 and $112 \mathrm{mg} / \mathrm{L}$ ) and were positive for PF4-antibody ELISA. In the analysis of the EudraVigilance database conducted by Krzywicka et al, ${ }^{18}$ the prevalence of prothrombotic risk factors in VITT cases was also considerably low (11\% in adenovirus-based COVID-19 vaccines recipients and $15 \%$ in those who received mRNAbased COVID-19 vaccines, compared with 64\% in CVT cases reported pre-COVID-19). Similarly, the use of oral contraceptives was nearly threefold lower (20 and $14 \%$ in recipients of adenovirus-based and mRNA-based COVID-19 vaccines, respectively) compared with the CVT cases reported preCOVID-19 (54\%).

Several other cases have since been described, as recently reviewed elsewhere. ${ }^{6}$ All of these shared similar aspects of being positive for anti-PF4 antibodies as measured by ELISA assays, but not with other immunological tests that are usually positive in patients with heparin-induced thrombocytopenia with thrombosis (HITT). Therefore, anti-PF4 antibodies are probably the main drivers of this life-threatening pathology, so that the presence of other (either mild or severe) pro-thrombotic factors does not additionally contribute to an otherwise already devastating thrombotic mechanism.

Beside the well-established role of anti-PF4 antibodies, the potential mechanisms underlying their generation after COVID-19, which may also encompass neutrophil activation and consequent release of the pro-thrombotic neutrophil extracellular traps (NETs), is still a matter of speculation ( $\mathbf{- F i g . 1} \mathbf{1}$ ).

Goldman and Hermans ${ }^{22}$ have speculated that, after intramuscular injection of adenoviruses-based COVID-19 vaccine, 
endothelial cells may be infected and induced to synthesize the spike protein of SARS-CoV-2, which could then bind with heparan sulfate proteoglycans at the luminal side or released by injured cells. To this end, the spike proteins could bind to angiotensin converting enzyme 2 (ACE2) at platelet surface, thus leading to their activation and PF4 release, which may then assume immunogenic properties after linkage with heparan sulfate proteoglycans shed from the same endothelial cells. Possible support to this theory was provided by studies showing that administration of adenovirus-based vaccines was effective to induce native-like post-translational processing and assembly of SARS-CoV-2 spike protein contained with the adenovirus vector. The spike protein is hence finally expressed at cell surface with the trimeric prefusion conformation capable to bind to its natural host receptors. ${ }^{23}$ The fact that COVID-19 vaccination may induce measurable amount of circulating spike protein, thus playing a possible role in the pathogenesis of VITT, is supported by data published by Ogata and colleagues. ${ }^{24}$

Another possible interpretation has been provided by Huynh and colleagues..$^{25}$ Briefly, it was first found that the binding of anti-PF4 antibodies in the sera of VITT patients involved eight surface amino acids of PF4, all located within the heparin binding site of the protein, but was different from the antigenic moiety recognized by the anti-PF4 antibodies developing in patients with HITT. These VITT anti-PF4 antibodies could instead effectively bind PF4, thus generating platelet-activating immune complexes, in the absence of heparin; this in turn would favor a cross-linking of FcrRIIa receptors on platelet surface, a phenomenon associated with activation of intracellular signaling events ultimately triggering platelet activation and aggregation. Another potential mechanism has been hypothesized by Kowarz and colleagues. ${ }^{26}$ Specifically, the authors postulated that adenovirus-based COVID-19 vaccines may trigger the transcription of wild-type and codon-optimized SARS-CoV-2 spike protein open reading frames. This would enable alternative splice events, generating C-terminal truncated and soluble SARSCoV-2 Spike protein variants, which may bind to ACE2expressing cells in the circulation, possibly platelets and endothelial cells, thus leading to their activation and thus finally favoring the generation of a pro-thrombotic milieu.

Irrespective of the precise mechanism, an important aspect in the pathogenesis of VITT is that virtually all patients with this syndrome have shown the presence of anti-PF4 antibodies. However, as analogous to heparin-induced thrombocytopenia (HIT), not all patients who develop antiPF4 antibodies after COVID-19 vaccination have thrombosis, as clearly shown in different published works. ${ }^{14,27}$ Interestingly, although the SARS-CoV-2 spike protein and PF4 share at least one similar epitope, the anti-PF4 antibodies found in VITT patients do not seemingly cross-reacted with SARSCoV-2 spike protein. ${ }^{27}$ It is hence conceivable that the association between anti-PF4 antibodies, thrombocytopenia and/or thrombosis may occur in a minority of predisposed patients, who may have some still unknown biological predisposition, or in those who develop a class of anti-PF4 antibodies that has larger platelet-activating potency. Last but not least, a direct interplay between the adenoviral vector and the platelets of the COVID-19 vaccine recipient cannot be ruled out, since it has been reported that some of these viruses can bind to platelet receptors (e.g., coxsackie and adenovirus receptor [CAR], CD46), thus triggering platelet activation, aggregation, and PF4 secretion. ${ }^{28}$ Importantly, it was also recently shown that although the development of anti-PF4 antibodies could be observed in most adenovirusbased AstraZeneca COPVID-19 vaccine recipients, these are non-platelet activating, are present in low titers and, last but not least, their serum level seems to correlate significantly with those of anti-SARS-CoV-2 neutralizing antibodies $(r=0.50 ; p<0.017) .^{29}$

It is also important to mention here that although all patients with CVT who tested positive for anti-PF4 antibodies in the study of Schulz et al had received the adenovirusbased AstraZeneca vaccine, ${ }^{14}$ none of those who received the Pfizer mRNA vaccine had a VITT risk score $>2$, meaning that the thrombotic events recorded in those who received Pfizer vaccine may represent another pathogenetic mechanism or may only be casually associated with vaccine administration, thus being perhaps more appropriately categorized as having vaccine-associated thrombosis (VAT) rather than VITT. To this end, it is noteworthy that although VITT seems to occur more commonly after receiving adenovirus-based COVID-19 vaccines, a limited number of individuals may also develop a form of acquired thrombotic thrombocytopenic purpura (TTP) after receiving mRNA vaccines, as also highlighted by Maayan et al. ${ }^{30}$ These cases are typically defined as being relatively young, and having considerably decreased platelet count, low ADAMTS-13 (a disintegrin and metalloprotease with a thrombospondin type 1 motif 13) activity and high anti-ADAMTS-13 antibody levels, thus confirming that different pathogenetic mechanisms may be involved in the socalled mRNA COVID-19 vaccines-associated thrombosis. The fact that the pathogenetic mechanisms involved in the cases of thrombocytopenia developing after adenovirus-based COVID-19 vaccination and those seen in COVID-19 mRNA vaccine recipients may be different has also been addressed by Lee et $\mathrm{al}^{15}{ }^{15}$ who described 20 patients (median age 41 years; $55 \%$ women) who developed secondary ITP after receiving either mRNA COVID-19 vaccine (i.e., 9 Pfizer and 11 Moderna). Fourteen of such patients (70\%) experienced significant hemorrhagic symptoms (bruising, petechiae, or mucosal bleeding) before hospitalization, while none developed clinically significant thrombosis.

\section{Characterization of Anti-PF4 Antibodies in VITT}

Since the pathogenesis of VITT is essentially based on development of anti-PF4 antibodies, their identification and quantification in plasma are essential for achieving a confirmation of the diagnosis. In this respect, is has been now clearly demonstrated that only ELISA-based anti-PF4 assays are able to identify the presence of such antibodies, since all the other automated commercial ("rapid") immunoassay display rather poor sensitivity. ${ }^{6}$ It is also important to 
highlight that not all ELISAs may reach $100 \%$ sensitivity in detecting these antibodies, as demonstrated by Platton et al. ${ }^{31}$ Therefore, when a single ELISA is negative in the presence of strong clinical suspicion, another test should be used to complement the results of the former. Clear evidence that ELISA methods are to be preferred for detecting anti-PF4 antibodies in patients with VITT has also been provided by a comprehensive report of the UK National External Quality Assessment Scheme, highlighting that all centers performing ELISAs reported positive result on both lyophilized and liquid VITT samples, while all centers using chemiluminescence, latex immunoassay, and lateral flow assays reported negative result on VITT lyophilized samples. ${ }^{32}$ Furthermore, an additional study published by Vayne and colleagues also found that the sensitivity of the methods for detecting anti-PF4 IgG antibodies depends on the antigen target, since significant levels of these antibodies could only be detected in the assays using PF4-poly(vinyl sulfonate) complex as antigenic target. $^{33}$ As an additional supplement to ELISA assays for anti-PF4 antibodies, consideration should also be given to performance of functional platelet activation assays. There exists a plethora of potential assays; however, some variable positivity may arise under the influence of technical test aspects. For example, the serotonin release assay can be modified by adding PF4 to increase diagnostic sensitivity. ${ }^{33}$ Furthermore, assay steps used in the assay to increase sensitivity to HITT, namely use of a therapeutic dose of heparin, seems to attenuate and sometimes even abolish the response in VITT. ${ }^{21}$ Many of these important technical aspects have been recently reviewed, ${ }^{6}$ where it was further stressed that the sensitivity of rapid chemiluminescent automated anti-PF4 immunoassays is indeed too low compared with that of more conventional ELISA techniques, so that only the latter should be used for this purpose. Yet, no ELISA method is like another and so, even among ELISA techniques, the diagnostic sensitivity may vary widely. 6,31 Another area of uncertainty is how long these antibodies will persist and whether or not they may be associated with an increased risk of thrombosis after receiving adenovirusbased vaccines for other infectious diseases. ${ }^{34}$

\section{Conclusion}

Unquestionable evidence has been provided about the binding of SARS-CoV-2 to platelets with a pathway that also involves the spike protein-ACE2 interaction, and that this binding is the followed by platelet activation, followed by necroptosis and apoptosis, ${ }^{35}$ while no similar evidence has been provided for the adenovirus-based AstraZeneca COVID-19 vaccine, in that challenge of citrate anticoagulated blood was not associated with platelet activation or aggregation, nor with binding of autologous antibodies to platelets. ${ }^{36,37}$ It is hence not really surprising that some forms of SARS-CoV-2 recombinant spike protein which were produced after human cells have been transfected by COVID-19 vaccination may enter the circulation and then trigger a similar cascade of events (i.e., platelet hyper-aggregation, which may then be followed by thrombosis, along with severe platelet injury, which may then cause thrombocytopenia) in a small proportion of vaccine recipients. Overall, VITT is a rare but potentially serious consequence of vaccination against COVID19 with certain adenovirus vaccines, namely AstraZeneca and Johnson and Johnson (Janssen). The most serious consequential thromboses are represented by CVT and splanchnic vein thrombosis. VITT represents "an immune response, leading to a condition similar to one seen sometimes in patients treated with heparin," that is HIT. ${ }^{38}$ However, there are some clinical and laboratory differences between VITT and HITT. Key to differentiating VITT and HITT is identification of prior heparin exposure, and laboratory testing using modifications of existing assays. For example, use of therapeutic heparin levels may augment both ELISA and functional testing (e.g., by serotonin release assay) in HITT, but would more typically lower ELISA and functional platelet responses in VITT. Also, rapid automated (e.g., AcuStar) plus ELISA anti-PF4 antibody tests would be positive in HITT, whereas in VITT only ELISA anti-PF4 antibody tests are typically positive.

Note

The opinions expressed in this review are those of the authors and do not necessarily represent the views of the University of Verona, Charles Sturt University, or NSW Health Pathology.

Funding

None.

Conflict of Interest

None declared.

\section{References}

1 Toor J, Echeverria-Londono S, Li X, et al. Lives saved with vaccination for 10 pathogens across 112 countries in a pre-COVID-19 world. eLife 2021;10:e67635

2 Evans SJW, Jewell NP. Vaccine effectiveness studies in the field. N Engl J Med 2021;385(07):650-651

3 Novak N, Tordesillas L, Cabanillas B. Adverse rare events to vaccines for COVID-19: from hypersensitivity reactions to thrombosis and thrombocytopenia. Int Rev Immunol 2021 (e-pub ahead of print). Doi: 10.1080/08830185.2021.1939696

4 Greinacher A, Thiele T, Warkentin TE, Weisser K, Kyrle PA, Eichinger S. Thrombotic thrombocytopenia after ChAdOx1 nCov-19 vaccination. N Engl J Med 2021;384(22):2092-2101

5 Schultz NH, Sørvoll IH, Michelsen AE, et al. Thrombosis and thrombocytopenia after ChAdOx1 $\mathrm{nCoV}-19$ vaccination. N Engl J Med 2021;384(22):2124-2130

6 Favaloro EJ. Laboratory testing for suspected COVID-19 vaccineinduced (immune) thrombotic thrombocytopenia. Int J Lab Hematol 2021;43(04):559-570

7 Lavin M, Elder PT, O'Keeffe D, et al. Vaccine-induced immune thrombotic thrombocytopenia (VITT) - a novel clinico-pathological entity with heterogeneous clinical presentations. Br J Haematol 2021 (e-pub ahead of print). Doi: 10.1111/bjh.17613

8 Lardinois B, Favresse J, Chatelain B, Lippi G, Mullier F. Pseudothrombocytopenia-a review on causes, occurrence and clinical implications. J Clin Med 2021;10(04):594

9 Hunter PR. Thrombosis after COVID-19 vaccination. BMJ 2021; 373(958):n958 
10 Pomara C, Sessa F, Ciaccio M, et al. Post-mortem findings in vaccine-induced thrombotic thombocytopenia. Haematologica 2021;106(08):2291-2293

11 Althaus K, Möller P, Uzun G, et al. Antibody-mediated procoagulant platelets in SARS-CoV-2-vaccination associated immune thrombotic thrombocytopenia. Haematologica 2021;106(08): 2170-2179

12 Simpson CR, Shi T, Vasileiou E, et al. First-dose ChAdOx1 and BNT162b2 COVID-19 vaccines and thrombocytopenic, thromboembolic and hemorrhagic events in Scotland. Nat Med 2021;27 (07):1290-1297

13 Cari L, Fiore P, Naghavi Alhosseini M, Sava G, Nocentini G. Blood clots and bleeding events following BNT162b2 and ChAdOx1 nCoV-19 vaccine: an analysis of European data. J Autoimmun 2021;122:102685

14 Schulz JB, Berlit P, Diener HC, et al;DGN SARS-CoV-2 Vaccination Study Group. COVID-19 vaccine-associated cerebral venous thrombosis in Germany. Ann Neurol 2021 (e-pub ahead of print). Doi: 10.1002/ana.26172

15 Lee EJ, Cines DB, Gernsheimer T, et al. Thrombocytopenia following Pfizer and Moderna SARS-CoV-2 vaccination. Am J Hematol 2021;96(05):534-537

16 Bikdeli B, Chatterjee S, Arora S, et al. Cerebral venous sinus thrombosis in the U.S. population, after adenovirus-based SARS-CoV-2 vaccination, and after COVID-19. J Am Coll Cardiol $2021 ; 78(04): 408-411$

17 Hwang J, Lee SB, Lee SW, et al. Comparison of vaccine-induced thrombotic events between ChAdOx1 nCoV-19 and Ad26.COV.2.S vaccines. J Autoimmun 2021;122:102681

18 Krzywicka K, Heldner MR, Sánchez van Kammen M, et al. PostSARS-CoV-2-vaccination cerebral venous sinus thrombosis: an analysis of cases notified to the European Medicines Agency. Eur J Neurol 2021 (e-pub ahead of print). Doi: 10.1111/ene.15029

19 Elalamy I, Gerotziafas G, Alamowitch S, et al;Scientific Reviewer Committee. SARS-CoV-2 Vaccine and Thrombosis: An Expert Consensus on Vaccine-Induced Immune Thrombotic Thrombocytopenia. Thromb Haemost 2021;121(08):982-991

20 Ulivi L, Squitieri M, Cohen H, Cowley P, Werring DJ. Cerebral venous thrombosis: a practical guide. Pract Neurol 2020;20(05): 356-367

21 See I, Su JR, Lale A, et al. US case reports of cerebral venous sinus thrombosis with thrombocytopenia after Ad26.COV2.S vaccination, March 2 to April 21, 2021. JAMA 2021;325(24):2448-2456

22 Goldman M, Hermans C. Thrombotic thrombocytopenia associated with COVID-19 infection or vaccination: possible paths to platelet factor 4 autoimmunity. PLoS Med 2021;18(05):e1003648

23 Watanabe Y, Mendonça L, Allen ER, et al. Native-like SARS-CoV-2 spike glycoprotein expressed by ChAdOx1 nCoV-19/AZD1222 vaccine. ACS Cent Sci 2021;7(04):594-602

24 Ogata AF, Cheng CA, Desjardins M, et al. Circulating SARS-CoV-2 vaccine antigen detected in the plasma of mRNA-1273 vaccine recipients. Clin Infect Dis 2021 (e-pub ahead of print). Doi: 10.1093/cid/ciab465
25 Huynh A, Kelton JG, Arnold DM, Daka M, Nazy I. Antibody epitopes in vaccine-induced immune thrombotic thrombocytopaenia. Nature 2021;596(7873):565-569

26 Kowarz E, Krutzke L, Reis J, Bracharz S, Kochanek S, Marschalek R. "Vaccine-induced Covid-19 mimicry" syndrome: splice reactions within the SARS-CoV-2 spike open reading frame result in spike protein variants that may cause thromboembolic events in patients immunized with vector-based vaccines. Res Sq 2021 (e-pub ahead of print). Doi: 10.21203/rs.3.rs-558954/v1

27 Greinacher A, Selleng K, Mayerle J, et al. Anti-platelet factor 4 antibodies causing VITT do not cross-react with SARS-CoV-2 spike protein. Blood 2021;138(14):1269-1277

28 Rzymski P, Perek B, Flisiak R. Thrombotic thrombocytopenia after COVID-19 vaccination: in search of the underlying mechanism. Vaccines (Basel) 2021;9(06):559

29 Terpos E, Politou M, Ntanasis-Stathopoulos I, et al. High prevalence of anti-PF4 antibodies following ChAdOx1 nCov-19 (AZD1222) vaccination even in the absence of thrombotic events. Vaccines (Basel) 2021;9(07):712

30 Maayan H, Kirgner I, Gutwein O, et al. Acquired thrombotic thrombocytopenic purpura: a rare disease associated with BNT162b2 vaccine. J Thromb Haemost 2021 (e-pub ahead of print). Doi: $10.1111 /$ jth. 15420

31 Platton S, Bartlett A, MacCallum P, et al. Evaluation of laboratory assays for anti-platelet factor 4 antibodies after ChAdOx1 nCOV19 vaccination. J Thromb Haemost 2021;19(08):2007-2013

32 Reilly-Stitt C, Kitchen S, Jennings I, et al. Anti-PF4 testing for vaccine-induced immune thrombocytopenia and thrombosis and heparin induced thrombocytopenia: results from a UK National External Quality Assessment Scheme exercise April 2021. J Thromb Haemost 2021 (e-pub ahead of print). Doi: 10.1111/ jth.15423

33 Vayne C, Rollin J, Gruel Y, et al. PF4 immunoassays in vaccineinduced thrombotic thrombocytopenia. N Engl J Med 2021;385 (04):376-378

34 Makris M, Pavord S, Lester W, Scully M, Hunt B. Vaccine-induced immune thrombocytopenia and thrombosis (VITT). Res Pract Thromb Haemost 2021;5(05):e12529

35 Koupenova M, Corkrey HA, Vitseva O, et al. SARS-CoV-2 initiates programmed cell death in platelets. Circ Res 2021 (e-pub ahead of print). Doi: 10.1161/CIRCRESAHA.121.319117

36 Limami Y, Khalki L, Zaid N, et al. Oxford-AstraZeneca ChAdOx1 COVID-19 vaccine does not alter platelet aggregation. Semin Thromb Hemost 2021 (e-pub ahead of print). Doi: 10.1055/s0041-1728831

37 Vinholt PJ, Frederiksen $\mathrm{H}$, Nielsen C. Modeling the effects of intravasal administration of AstraZeneca ChAdOx1 nCoV-19 vaccine on human platelets. Thromb Haemost 2021;121(12): 1681-1683

38 Nazy I, Sachs UJ, Arnold DM, et al. Recommendations for the clinical and laboratory diagnosis of VITT against COVID-19: Communication from the ISTH SSC Subcommittee on Platelet Immunology. J Thromb Haemost 2021;19(06):1585-1588 